\title{
Solitary fibrous tumors of the pleura: Results of surgical treatment and long-term prognosis
}

\author{
Karen M. Harrison-Phipps, MD, ${ }^{\mathrm{a}}$ Francis C. Nichols, MD, ${ }^{\mathrm{a}}$ Cathy D. Schleck, BS, ${ }^{\mathrm{b}}$ \\ Claude Deschamps, MD, ${ }^{\mathrm{a}}$ Stephen D. Cassivi, MD, MSc, ${ }^{\mathrm{a}}$ Paul H. Schipper, MD, ${ }^{\mathrm{a}}$ Mark S. Allen, MD, ${ }^{\mathrm{a}}$ \\ Dennis A. Wigle, $\mathrm{MD}, \mathrm{PhD},{ }^{\mathrm{a}}$ and Peter C. Pairolero, $\mathrm{MD}^{\mathrm{a}}$
}

Objective: We sought to define the long-term outcome of surgically treated solitary fibrous tumors of the pleura.

Methods: We performed a retrospective review from December 1972 through December 2002.

\begin{abstract}
Results: There were 84 patients (39 men and 45 women) with a median age of 57 years (range, 34-83 years). Forty-six patients were symptomatic. Surgical resection included pulmonary wedge excision in 62 patients, lobectomy in 4 patients, segmentectomy in 2 patients, chest wall resection in 3 patients, isolated pleural resection in 7 patients, and chest wall resection with pulmonary wedge excision, lobectomy, or pneumonectomy in 3 , 2, and 1 patients, respectively. Tumors were polypoid in 57 patients, sessile in 20 patients, and intrapulmonary in 7 patients. Histopathology was benign in 73 and malignant in 11 patients. Nine $(82 \%)$ patient with malignant tumors and $37(54 \%)$ patients with benign tumors were symptomatic $(P=.11)$. The median tumor diameters for malignant and benign tumors were 12.0 and $4.5 \mathrm{~cm}$, respectively $(P=.001)$. Operative mortality and morbidity occurred in $3(3.6 \%)$ and $7(8.1 \%)$ patients, respectively. Median follow-up in survivors was 146 months (range, 23-387 months). Median survival for patients with benign and malignant tumors was 284 and 55 months, respectively, and 5-year survival was $88.9 \%$ and $45.5 \%$, respectively $(P=.0005)$. Eight $(9.5 \%)$ patients had recurrent solitary fibrous tumors of the pleura. Recurrences were malignant in 6 and benign in 2 patients. Localized chest recurrences occurred in 3 patients, all of whom had reresection, with 2 patients again having recurrence.
\end{abstract}

Conclusion: Resection of benign solitary fibrous tumors of the pleura carries an excellent prognosis. Larger tumors are more likely to be malignant. Both benign and malignant tumors can recur. Although prolonged survival after resection of malignant tumors is possible, recurrence is common.

Solitary fibrous tumors of the pleura (SFTPs) are rare mesenchymal tumors most commonly arising in the pleura. They can, however, also present as an intrapulmonary mass. ${ }^{1}$ SFTPs are distinct from diffuse malignant mesothelioma (DMM), which is associated with asbestos exposure and typically carries a poor prognosis. ${ }^{2}$ The histopathologic characteristics of SFTPs were first described by Klemperer and Rabin in $1931 .^{3}$ Over the years, SFTPs have been characterized by a number of terms, including fibrous mesothelioma, localized fibrous mesothelioma, localized mesothelioma, localized fibrous tumors, fibrous mesothelioma, solitary fibrous mesothelioma, pleural fibroma, submesothelial fibroma, and subserosal fibroma. ${ }^{4}$ The absence of clear nomenclature has only added to the confusion about these tumors,

\footnotetext{
From the Division of General Thoracic Surgery ${ }^{a}$ and the Section of Biostatistics, ${ }^{b}$ Mayo Clinic, Rochester, Minn.

Read at the Eighty-seventh Annual Meeting of the American Association for Thoracic Surgery, Washington, DC, May 5-9, 2007.

Disclosures: Dr Stephen D. Cassivi reports consulting fees from Spiration Inc.

Received for publication May 5, 2007; revisions received Dec 17, 2008; accepted for publication Jan 13, 2009.

Address for reprints: Francis C. Nichols, MD, Division of General Thoracic Surgery, Mayo Clinic, 200 First St SW, Rochester, MN 55905 (E-mail: nichols.francis@ mayo.edu).

J Thorac Cardiovasc Surg 2009;138:19-25

$0022-5223 / \$ 36.00$

Copyright $\subset 2009$ by The American Association for Thoracic Surgery

doi:10.1016/j.jtcvs.2009.01.026
}

and uncertainty exists with regard to their clinical behavior. The purpose of this report is to review our experi-ence with patients who underwent surgical resection for SFTPs.

\section{MATERIALS AND METHODS}

Between December 1972 and December 2002, 84 patients underwent surgical treatment for SFTPs at the Mayo Clinic in Rochester, Minnesota, and these patients formed the basis for this review. The study was approved by the Mayo Foundation's Institutional Review Board. Medical records were analyzed for age, sex, signs and symptoms, radiographic findings, tumor histopathology, location, surgical resection, clinical outcome, and survival. Operative mortality included patients who died within the first 30 days after surgical intervention or during the same hospital admission. Follow-up was obtained from retrospective review of the patients' medical records and, where necessary, written surveys. Malignant SFTPs were diagnosed when more than 4 mitoses per 10 high-power fields were seen on light microscopic analysis. ${ }^{5}$ Additional microscopic supportive features of malignancy included areas of hemorrhage and necrosis, areas of high cellularity with crowding and overlapping nuclei, marked pleomorphism, and vascular invasion. ${ }^{5}$ The probability of survival and the cumulative probability of recurrence were estimated by using the Kaplan-Meier survivorship method. ${ }^{6}$ Recurrence and survival were estimated starting at the date of the first SFTP resection and are reported with $95 \%$ confidence intervals (CIs). Survival curves of the patients were compared with expected curves of persons of the same age and sex, as derived from vital statistics for the west-north-central region of the United States. ${ }^{7}$ Comparisons of survivorship curves were made with 1 -sample log-rank tests. ${ }^{8}$ Comparisons of discrete variables between benign and malignant pathology were made with Fisher's exact tests. ${ }^{9}$ Comparisons of continuous variables between benign 

Abbreviations and Acronyms
$\mathrm{CI}=$ confidence interval
$\mathrm{CT}=$ computed tomography
DMM $=$ diffuse malignant mesothelioma
FNA $=$ fine-needle aspiration
PET = positron emission tomography
SFTP $=$ solitary fibrous tumor of the pleura
VATS $=$ video-assisted thoracic surgery

and malignant pathology were made with Wilcoxon rank sum tests. ${ }^{10}$ All statistical tests were 2 -sided.

\section{RESULTS}

There were 45 women and 39 men with a median age of 57 years (range, 34-83 years) enrolled in the study. Forty-five (54\%) patients had a positive history of cigarette smoking. Median pack-years were 30 (range, 1-100 packyears). Forty-six $(55 \%)$ patients were symptomatic, with chest pain in $15(18 \%)$, cough in $8(9.5 \%)$, dyspnea in $3(3.6 \%)$, and various other symptoms in $12(14 \%)$. Eight $(9.5 \%)$ patients had multiple symptoms. Extrathoracic symptoms, such as hypertrophic pulmonary osteoarthropathy alone in $3(3.6 \%)$ patients, hypertrophic pulmonary osteoarthropathy and digital clubbing in $5(6.0 \%)$ patients, and hypoglycemia in $1(1.2 \%)$ patient, were present. Chest radiographs were obtained for the entire study group, and the SFTPs were identified in $77(92 \%)$ patients. Before use of computed tomographic (CT) analysis, conventional chest tomographic scans were obtained in $15(18 \%)$ patients, and the SFTPs were visualized in all. Sixty-two (74\%) patients had CT scans of the chest, with the SFTPs visualized in 61 $(98 \%)$. Magnetic resonance imaging was obtained in 4 $(4.8 \%)$ patients, and results were abnormal in all. The SFTPs occurred on the right side in $50(60 \%)$ patients and the left side in $34(40 \%)$ patients. CT-guided percutaneous biopsies were performed in $22(26 \%)$ patients and were diagnostic in $10(45 \%)$ patients. Transbronchial biopsies were performed in $3(3.6 \%)$ patients and were diagnostic in $1(33 \%)$ patient.

All patients underwent surgical treatment. Postoperative chemotherapy and chemoradiotherapy were administered to 1 patient each. The operative approach was a posterolateral thoracotomy in $68(81 \%)$ patients, video-assisted thoracic surgery (VATS) in $12(14 \%)$ patients, a sternotomy in $3(3.6 \%)$ patients, and a transabdominal approach in 1 $(1.2 \%)$ patient. The transabdominal approach was used for a paraesophageal hiatal mass, which at the time of surgical intervention proved to be an SFTP that was able to be resected through this approach. The SFTPs were polypoid in $57(68 \%)$ patients, sessile in $20(24 \%)$ patients, and intrapulmonary in $7(8.3 \%)$ patients. At the time of the operation, the SFTPs appeared to arise from the visceral pleura in 58 $(69 \%)$ patients, the parietal pleura in $15(18 \%)$ patients, and both in $4(4.8 \%)$ patients and were intrapulmonary without obvious visceral pleural involvement in $7(8.3 \%)$ patients.

Surgical resection was pulmonary wedge excision in 62 $(74 \%)$ patients, lobectomy in $4(4.8 \%)$ patients, segmentectomy in $2(2.4 \%)$ patients, isolated parietal pleural resection alone in $7(8.3 \%)$ patients, chest wall resection alone in 3 $(3.6 \%)$ patients, and chest wall resection combined with concomitant pulmonary wedge excision, lobectomy, or pneumonectomy in 3, 2, and 1 patients, respectively. SFTP resection was complete in all patients. SFTPs were benign in $73(87 \%)$ patients and malignant in $11(13 \%)$ patients. Nine $(82 \%)$ patients with malignant SFTPs and 37 (54\%) patients with benign SFTPs were symptomatic $(P=.11)$. Morphologically benign SFTPs were polypoid, sessile, and intraparenchymal in $51(70 \%), 15(20 \%)$, and $7(10 \%)$ patients, respectively. Similarly, malignant SFTPs were polypoid and sessile in 4 (36\%) and 7 (64\%) patients, respectively. Morphologic and pleural characteristics of the SFTPs are shown in Table 1. Neither the morphologic characteristics nor the site of pleural origin aided in the differentiation of benign from malignant SFTPs $(P=.08)$. The median overall tumor diameter was $5 \mathrm{~cm}$ (range, $0.8-30 \mathrm{~cm}$ ). The median diameter was $12 \mathrm{~cm}$ (range, $7-19 \mathrm{~cm}$ ) for malignant SFTPs and $4.5 \mathrm{~cm}$ (range, $0.8-30 \mathrm{~cm}$ ) for benign SFTPs $(P=.001)$. In $7(64 \%)$ of the 11 patients with malignant SFTPs, the tumor diameter was greater than $10 \mathrm{~cm}$, whereas the tumor was greater than $10 \mathrm{~cm}$ in only $17(23 \%)$ of the 73 patients with benign SFTPs $(P=.01)$.

Mediastinal lymphadenectomy or lymph node sampling was performed at the individual discretion of the surgeon in $11(13 \%)$ patients. Four of these patients had recurrent SFTPs, and 6 had malignant tumors. Mediastinal lymph node removal was undertaken on larger SFTPs with a median diameter of $7.5 \mathrm{~cm}$ (range, 1.5-19 cm). On histopathologic examination, no tumor was found in any of the lymph nodes. Complications occurred in $9(11.8 \%)$ patients and included atrial fibrillation, bleeding, and reoperation for bleeding in 2 $(2.4 \%)$ patients each and pleural effusion, pneumonia, and postoperative ileus in $1(1.2 \%)$ patient each.

Three patients died (operative mortality, 3.6\%). One patient with no prior history of coronary artery disease

TABLE 1. Characteristics of solitary fibrous tumors of the pleura

\begin{tabular}{|c|c|c|c|c|}
\hline & $\begin{array}{c}\text { Benign, } \\
\text { no. of } \\
\text { patients }(\%)\end{array}$ & $\begin{array}{c}\text { Malignant, } \\
\text { no. of } \\
\text { patients }(\%)\end{array}$ & $P$ value* & $\begin{array}{c}\text { Recurrence, } \\
\text { no. of } \\
\text { patients }\end{array}$ \\
\hline Morphology & & & .17 & \\
\hline Polypoid & $51(70)$ & $6(55)$ & & $5(88)$ \\
\hline Sessile & $14(19)$ & $5(45)$ & & $3(16)$ \\
\hline Intrapulmonary & $8(11)$ & 0 & & \\
\hline Pleura & & & .08 & \\
\hline Parietal & $12(16)$ & $3(27)$ & & $2(13)$ \\
\hline Visceral & $51(70)$ & $6(55)$ & & $3(53)$ \\
\hline Both & $2(3)$ & $2(18)$ & & $3(75)$ \\
\hline Intrapulmonary & $8(11)$ & 0 & & \\
\hline
\end{tabular}


died suddenly on postoperative day 3 of a myocardial infarction. The second patient had known renal insufficiency and severe atherosclerosis and died on postoperative day 9 of mesenteric ischemia. The final patient had been discharged without complication from the hospital on postoperative day 7 and died of unknown cause on postoperative day 12 at home.

Follow-up was complete in all survivors and ranged from 2 to 32 years (median, 12 years). At last follow-up, 56 patients were alive, and 27 had died. The overall 5-year survival was $83 \%$ $(95 \%$ CI, $75 \%-92 \%$ ), with a median survival of 284 months and an expected survival of $91 \%(P=.19$, Figure 1). Median survival for malignant SFTPs was 55 versus 284 months for benign SFTPs. Five-year survival for benign and malignant SFTPs was $89 \%(95 \%$ CI, $82 \%-96 \%)$ and $45.5 \%(95 \%$ CI, 24\%-87\%), respectively ( $P=.0005$, Figure 2$)$.

Overall, 5-year freedom from recurrence was $95 \%(95 \%$ CI, $90 \%-100 \%)$. Of 81 operative survivors, $8(9.9 \%)$ patients experienced recurrent SFTPs. For patients experiencing recurrence, the surgical approach was a thoracotomy in 7 patients and a median sternotomy in 1 patient. The site of pleural origin for recurrent SFTPs was visceral in 4 patients, parietal in 2 patients, and both in 2 patients. Five-year freedom from recurrence for patients with benign and malignant SFTPs was $100 \%$ and $58 \%$ (95\% CI, 34\%-100\%), respectively $(P<.0001$, Figure 3$)$. Ultimately, $6(75 \%)$ patients with malignant SFTPs and $2(25 \%)$ patients with benign SFTPs experienced recurrence. Median time to recurrence for malignant and benign SFTPs was 34 and 83 months, respectively. In the 6 patients with initially malignant SFTPs that recurred, the recurrences were malignant in 5 patients and benign in 1 patient. In the 2 patients with benign SFTPs that recurred, the recurrence was benign and malignant in $1 \mathrm{pa}-$ tient each. Recurrences were localized in $3(37.5 \%)$ patients and widely metastatic in $5(62.5 \%)$ patients. All 3 patients

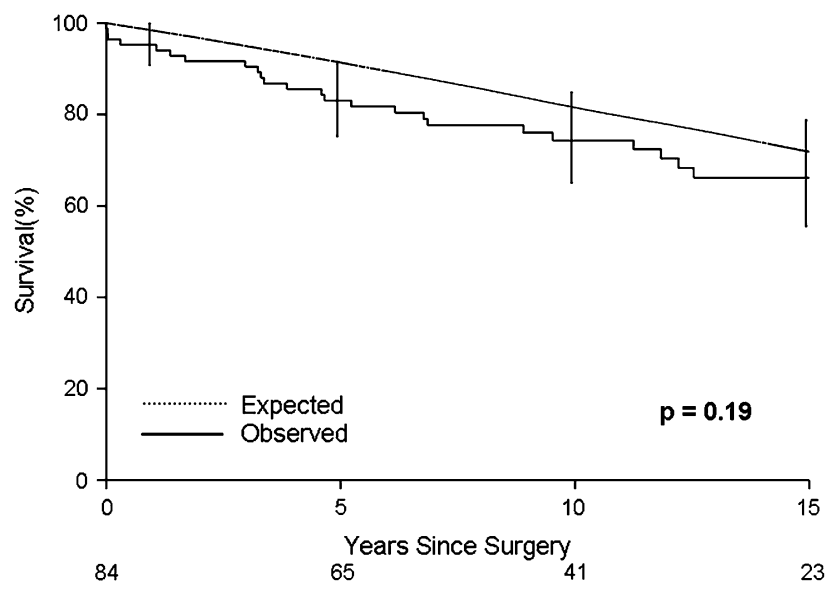

FIGURE 1. Overall survival (death from any cause) of 84 patients undergoing resection of solitary fibrous tumors of the pleura. Zero time on the abscissa is the date of the operation. Numbers of patients at risk are shown below the abscissa.

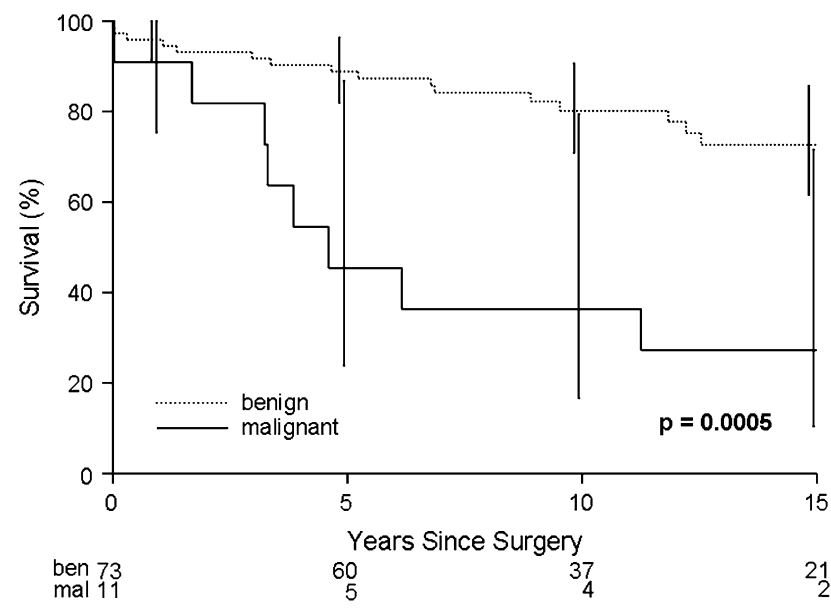

FIGURE 2. Survival (death from any cause) in 73 patients who underwent resection of benign solitary fibrous tumors of the pleura compared with that in 11 patients who had resection of malignant solitary fibrous tumor. Zero time on the abscissa is the date of the operation. Numbers of patients at risk are shown below the abscissa.

with localized recurrences underwent reresection, and 2 STFPs again recurred. The SFTP recurrence was diffusely metastatic within the abdominal cavity and localized to the ipsilateral hemithorax in 1 patient each. The latter patient eventually underwent a total of 4 resections over a 24-year period, after which he remained without evidence of disease. In the 5 patients with widely metastatic recurrent SFTPs, the observed sites of distant metastases included bone, contralateral hemithorax, liver, peritoneum, and retroperitoneum.

\section{DISCUSSION}

SFTPs are uncommon neoplasms, with just over 900 cases reported in the literature. ${ }^{4,11}$ Although SFTPs were

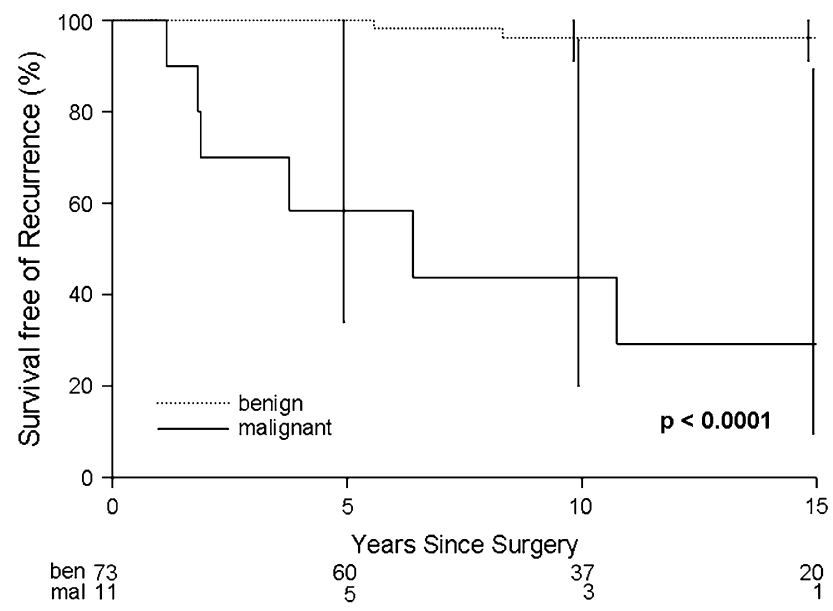

FIGURE 3. Survival (death from any cause) free of recurrence in 73 patients who underwent resection of benign solitary fibrous tumors of the pleura compared with that in 11 patients who had resection of malignant solitary fibrous tumors. Zero time on the abscissa is the date of the operation. Numbers of patients at risk are shown below the abscissa. 
TABLE 2. Immunohistochemical profile of SFTPs and diffuse malignant mesothelioma

\begin{tabular}{lccccc}
\hline Tumor & Cytokeratin & Vimentin & CD34 & CD99 & Bcl-2 \\
\hline SFTP & - & + & + & + & + \\
DMM & + & - & - & - & - \\
\hline
\end{tabular}

SFTP, Solitary fibrous tumor of the pleura; -, negative; +, positive; DMM, diffuse malignant mesothelioma.

historically considered benign tumors, Okike and colleagues, ${ }^{12}$ from our institution, in 1978 emphasized their malignant potential. Nevertheless, because of a lack of understanding of the cellular origins of SFTPs and their seemingly indolent clinical course, many physicians still consider SFTPs to be a benign variant of mesothelioma. ${ }^{13}$

Localized mesothelioma of the pleura was first described by Wagner in $1870 .{ }^{12}$ In 1931, Klemperer and Rabin ${ }^{3}$ distinguished localized mesothelioma from diffuse mesothelioma and proposed submesothelial cells as the cellular origin for the localized variety. Controversy developed in 1941, when Stout and Murray ${ }^{14}$ claimed mesothelial cells to be the cellular origin of localized mesothelioma. More recently, immunohistochemical techniques, flow cytometric studies, and electron microscopic analysis have demonstrated that SFTPs indeed arise from the mesenchymal cells beneath the mesothelial lining of the pleura, as initially postulated by Klemperer and Rabin. ${ }^{2-4,11,15,16}$ This mesothelial versus mesenchymal difference in cell origin is likely key to the wide dissemination of DMM over serosal surfaces, eventually leading to organ encasement, in contrast to the more localized spread of SFTPs. Based on this differentiation in cellular origin, the term localized mesothelioma has been abandoned in favor of SFTPs. ${ }^{17}$ SFTPs have no known association with asbestos exposure. DMM, unlike SFTPs, has a poor clinical course, with death often occurring within 2 years of diagnosis. Immunohistochemistry is a useful adjunct in the differentiation of SFTPs from DMM. SFTPs, in distinction to DMM, stain positive for vimentin and CD34 and negative for keratin. Table 2 outlines the distinct immunohistochemical profile of SFTPs and DMM.

Our series, like others, show SFTPs to most commonly arise in the sixth decade of life. Although some authors have stated that SFTPs are seen equally in men and women, ${ }^{4,15}$ our large series and others ${ }^{11,12,18}$ show a predilection for women. Approximately half of patients are symptomatic on presentation, and symptoms are more common in patients with malignant SFTPs. Common symptoms include chest pain and cough. Extrathoracic symptoms, such as hypertrophic pulmonary osteoarthropathy, clubbing, and hypoglycemia, have been reported in up to $66 \%$ of patients ${ }^{12,19,20}$ but occurred in only $9(11 \%)$ of our patients. This frequency of symptoms is less than both the $66 \%$ and $19 \%$ frequencies reported in our earlier series ${ }^{12,19}$ but still greater than the frequencies reported in most recent series from other institutions. ${ }^{11,16,18}$ The extrathoracic symptoms

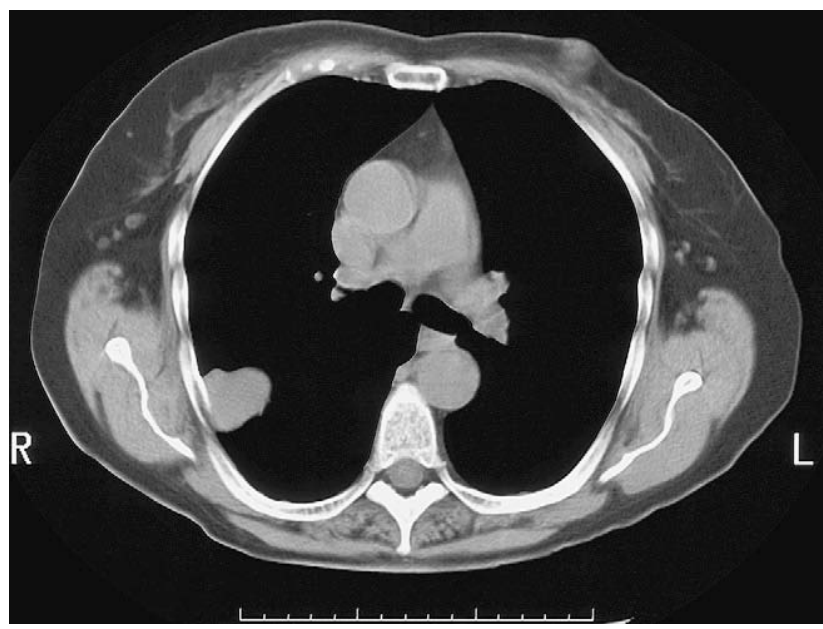

FIGURE 4. Computed tomographic scan showing a small solitary fibrous tumor of the visceral pleura.

resolved in all of our patients after surgical resection. Chest CT scanning is the radiographic test of choice, often showing a well-circumscribed homogenous mass of soft tissue attenuation; however, this finding lacks specificity, and heterogeneity is possible (Figures 4 and 5). The tumors commonly show displacement of surrounding structures, although some might appear as intraparenchymal masses. CT scanning cannot differentiate benign from malignant SFTPs. The diagnostic accuracy of CT-guided biopsy was $45 \%$ in our series and thus, like other series, is considered unacceptably low. ${ }^{13,16,18,21}$ Because of these variable results, we do not routinely recommend CT-guided biopsy. CT-guided biopsy would be considered in patients at too high a risk for surgical resection or patients with radiographically unresectable masses to try and obtain a histopathologic diagnosis.

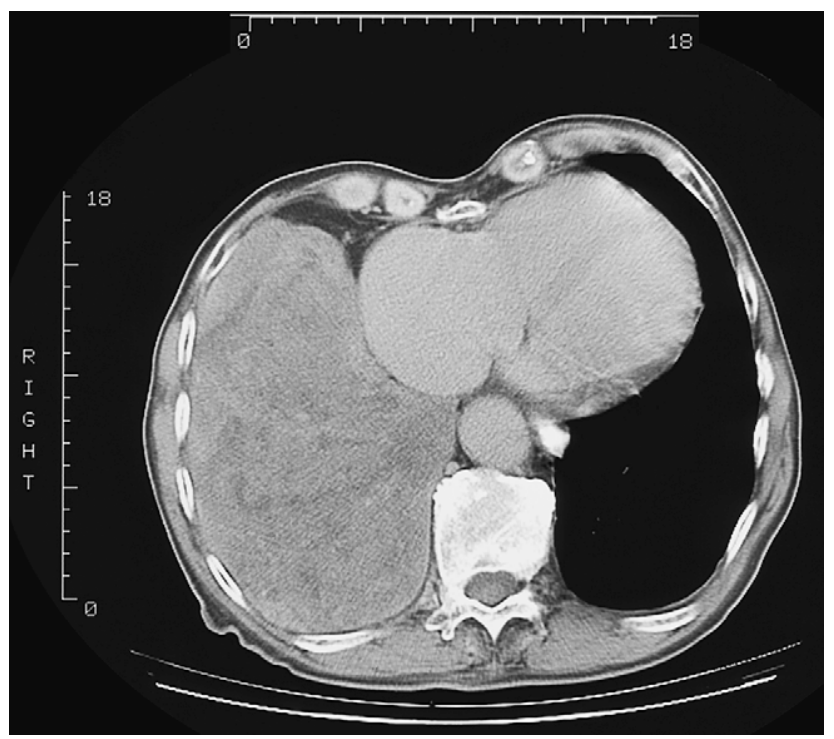

FIGURE 5. Computed tomographic scan showing a giant solitary fibrous tumor of the pleura. It appears somewhat inhomogeneous. 
Complete surgical resection is the preferred treatment for SFTPs. VATS was used in only $14 \%$ of our patients, but this clearly reflects the 30-year time span of this series, with VATS being available only in the latter 15 years. The median SFTP size for VATS resections was $2.4 \mathrm{~cm}$ (range, $0.8-15 \mathrm{~cm}$ ), and there were no recurrences. The median SFTP size for open resections was $7 \mathrm{~cm}$ (range, 1-30 cm). Pulmonary wedge excision was the most common surgical procedure, occurring in nearly three quarters of our patients, and formal anatomic lung parenchymal resections alone were performed in only $6(7.1 \%)$ patients. Chest wall resection alone was required in $3(3.6 \%)$ patients and was associated with an additional lung parenchymal resection in 6 more patients. The operative approach and extent of surgical excision should be dictated by the SFTP's size and location. SFTPs in our series ranged in size from 0.8 to $30 \mathrm{~cm}$, with a median diameter of $5 \mathrm{~cm}$. Size was the best clinical predictor of malignancy, with malignant tumors having a median diameter of $12 \mathrm{~cm}$ in comparison with $4.5 \mathrm{~cm}$ in benign tumors $(P=.001)$.

Long-term survival after resection of both benign and malignant SFTPs is possible, with the overall median survival in our series being 24 years. However, long-term survival for completely resected benign SFTPs is dramatically better than that of completely resected malignant SFTPs (Figure 2). Long-term follow-up after complete resection is necessary because SFTPs can recur, and this was seen in $8(9.9 \%)$ of our patients, including 2 patients with benign histopathology. Several authors have not reported recurrence after complete resection of benign SFTPs. ${ }^{11,13,16,18,21}$ In contrast, reported recurrence rates for completely resected malignant SFTPs range from $14 \%$ to $86 \% .{ }^{11,13,16,18}$ In our series there were 10 surviving operative patients with malignant SFTPs, and $6(75 \%)$ of these experienced recurrence. In only 3 patients ( 2 with benign SFTPs and 1 with a malignant SFTP) was the recurrence localized to the same hemithorax. After reoperation, recurrence again occurred in 2 patients.

In conclusion, SFTPs are uncommon tumors that are best treated with complete surgical resection. In this series increased tumor size is suggestive of malignancy. Longterm survival after resection of benign SFTPs is excellent but is also possible after resection for malignant SFTPs. However, malignant SFTPs are prone to recurrence, which in turn is associated with a poor prognosis.

\section{References}

1. Wilson R, Fishback N, Colby T, Fleming M, Koss M, Travis W. Intrapulmonary localized fibrous tumor (LFT): a clinicopathologic analysis of 43 cases [abstract]. Mod Pathol. 1995;8:155A.

2. Flores RM, Rusch VW. Diffuse malignant mesothelioma. In: Shields TW, Locicero J, Ponn RB, Rusch VW, eds. General thoracic surgery. 6th ed. Philadelphia: Lippincott Williams \& Wilkins; 2005. p. 917.

3. Klemperer P, Rabin CB. Primary neoplasms of the pleura. A report of five cases. Arch Pathol. 1931;11:385-412.
4. de Perrot M, Fischer S, Bründler MA, Sekine Y, Keshavjee S. Solitary fibrous tumors of the pleura. Ann Thorac Surg. 2002;74:285-93.

5. England DM, Hochholzer L, McCarthy MJ. Localized benign and malignant fibrous tumors of the pleura-a clinico-pathological review of 223 cases. Am J Surg Pathol. 1989;13:640-58.

6. Kaplan EL, Meier P. Non-parametric estimation from incomplete observations. J Am Stat Assoc. 1958;53:457-81.

7. Bergstrahl EJ, Offord KP. Conditional probabilities used in calculating cohort expected survival. Rochester (MN): Section of Biostatistics, Mayo Clinic; 1988. Technical Report Series No. 37.

8. Peto R, Peto J. Asymptotically efficient rank invariant procedures. J R Stat Soc [A]. 1972;135:185-206.

9. Snedecor GW, Cochran WG. In: Statistical methods. 6th ed. Ames (IA): Iowa State University Press; 1967. p. 100-6.

10. Dixon WJ, Massey F. In: Introduction to statistical analysis. 3rd ed. New York: McGraw Hill; 1969. p. 77-80, 116-8, 156-63, 344-5, 509.

11. Sung SH, Chang JW, Kim J, Lee KS, Han J, Park S. Solitary fibrous tumors of the pleura: Surgical outcome and clinical course. Ann Thorac Surg. 2005;79:303-7.

12. Okike N, Bernatz PE, Woolner LB. Localized mesothelioma of the pleura. Benign and malignant variants. J Thorac Cardiovasc Surg. 1978;75:363-72.

13. Santos RS, Haddad R, Lima CE, Liu YL, Misztal M, Ferreira TD, et al. Patterns of recurrence and long-term survival after curative resection of localized fibrous tumors of the pleura. Clin Lung Cancer. 2005;7:197-201.

14. Stout AP, Murray MR. Localized pleural mesothelioma. Investigation of its characteristics and histogenesis by the method of tissue culture. Arch Pathol. 1942;34: 951-64.

15. Mitchell JD. Solitary fibrous tumor of the pleura. Semin Thorac Cardiovasc Surg. 2003;15:305-9.

16. Cardillo G, Facciolo F, Cavazzana AO, Capece G, Gasparri R, Martelli M. Localized (solitary) fibrous tumors of the pleura: an analysis of 55 patients. Ann Thorac Surg. 2000;70:1808-12.

17. Chan JKC. Solitary fibrous tumour-everywhere, and a diagnosis in vogue. His topathogy. 1997;31:568-76.

18. Magdeleinat P, Alifano M, Petino A, LeRochais JP, Dulmet E, Galateau F, et al Solitary fibrous tumors of the pleura: clinical characteristics, surgical treatment and outcome. Eur J Cardiothorac Surg. 2002;21:1087-93.

19. Clagett OT, McDonald JR, Schmidt HW. Localized fibrous mesothelioma of the pleura. J Thorac Surg. 1952;24:213-30.

20. Briselli M, Mark EJ, Richard DG. Solitary fibrous tumors of the pleura: eight new cases and review of 360 cases in the literature. Cancer. 1981;47:2678-89.

21. de Perrot M, Kurt AM, Robert JH, Borisch B, Spiliopoulos A. Clinical behavior of solitary fibrous tumors of the pleura. Ann Thorac Surg. 1999;67:1456-9.

\section{Discussion}

Dr Robert J. Downey (New York, NY). Karen, I enjoyed that very much.

SFTPs are going to be seen by all of us, but sporadically, such that it is for most surgeons to get a sense for the disease. Therefore I enjoy articles like this that flesh out their behavior based on large institutional experiences.

I have 2 comments and 3 questions. My first comment is that when I went over the data, it was clear I thought this would be an excellent opportunity with a large data set of pathologic material to re-examine the pathologic criteria for malignancy. I think that your data validate England and colleagues' data from 1989, which established criteria for separating benign from malignant tumors in that those criteria accurately predicted those patients of yours who were likely to have recurrence and those who were not.

My second comment is that you noted that fine-needle aspiration (FNA) only yielded a diagnosis in about $45 \%$ of patients, and the article suggests that you do not recommend performing FNA before surgical intervention. I would suggest that your study is not designed to address this question because you do not know the denominator of the patients with solitary pulmonary nodules who underwent needle biopsy. Diagnoses of other types of tumors might 
have been made in other patients. Your data simply suggest that if you are eventually going to know that this tumor is a solitary fibrous tumor, then you should not do a needle biopsy up front.

My questions are as follows. First, when these lesions are small, operations are actually very easy. The large ones can be extremely difficult, in that they can be hard to move around the chest, such that you can have trouble finding the point of attachment, which is clearly important for adequacy of resection. They can be hard to remove through an incision when they get up to 15 and $25 \mathrm{~cm}$ in size, and they can be so large that they press against the boney structures of the chest wall, develop venous engorgement, and bleed until the chest is widely opened. You state only that these patients underwent thoracotomies and sternotomies. Could you describe what extended approaches to the thorax, such as a hemiclamshell, you have found useful in an attempt to achieve better exposure?

Second, some of the previous articles on benign fibrous tumors have suggested that benign lesions can undergo malignant degeneration. Your data do not support this in that you saw only 1 patient who had a recurrence that was read as malignant where the original site of disease had been believed to be benign. To me, 1 transformation event in about 80 patients suggests that the problem is with the adequacy of pathology, which in these other series might not have been complete. These could be heterogeneous tumors, and if you look carefully enough, you will eventually see the malignant components.

Finally, based on the very poor outcomes seen in the patients with malignant disease, are you recommending adjuvant therapy once the resections have been completed?

Thank you.

Dr Harrison-Phipps. Thank you very much, Dr Downey, for your comments and questions.

If I could just address one of your comments first, regarding the FNA cytology, you correctly said that this had a positive predictive value in $45 \%$ of our patients or that the sensitivity, rather, was correct in $45 \%$ of the patients, and this is pretty low. If you look through a number of the series, the large series, in the majority of them, it is really the minority of patients who have had a preoperative FNA, but in no case series is the sensitivity higher than $45 \%$, and in some it is lower. I would say that there is 1 article that mentions that perhaps using a wide-bore, Tru-Cut biopsy needle might result in improved diagnostic accuracy, but that was a small study with only 5 patients. I think the problem that occurs is that it is difficult to decide whether they are benign or malignant solitary fibrous tumors, and this is simply, as you said, because of the heterogeneity of the tumors. It would be easy to get an incorrect or a sampling error.

Regarding the large tumors, in our series about a third of tumors were larger than $15 \mathrm{~cm}$, and in all our patients the resection was complete. That is the first thing. When you look through the majority of the case series, in fact, the majority of authors support the use of a thoracotomy for resection of these tumors, and certainly there are isolated case reports that describe a number of operative approaches for massive tumors, including hemiclamshell and so on. We did use sternotomy in 3 patients, but, interestingly, those were actually all for tumors of less than $10 \mathrm{~cm}$, and it was just their location and not the size that prompted the choice of this incision. Therefore what I would say is that I truly advocate the use of the hemiclamshell incision. I think it is an excellent incision with great exposure. It is just that we did not find it necessary in our series. Our largest tumor was $30 \mathrm{~cm}$, and in all cases all tumors were removed through an extended posterolateral thoracotomy, if necessary.

Regarding the transformation from benign to malignant, there was only 1 such patient in our series. We did go back and ask the pathologists to review the slides, and they confirmed that in fact the original pathology was benign and the recurrence was malignant. Various case series report no changes. I think the problem is that these tumors are such a mixed bag, it really depends what you are going to get. In some series the incidence is higher. I just really think they are very individual tumors.

Regarding your question on adjuvant or neoadjuvant therapies, chemotherapy or radiotherapy, there really are no data to make any legitimate comment. There are certainly isolated comments within oncology articles, and one in particular mentioned a patient who had positive resection margins with an almost inoperable tumor, an unresectable tumor. This patient had radiotherapy that shrunk the tumor and was alive 20 years later. That was a benign tumor. In another case a patient received adriamycin and isophosphamide and experienced some tumor shrinkage. But really, there are no real data to support the use or choice of any regimen at all.

Dr P. Michael McFadden (Los Angeles, Calif). Dr HarrisonPhipps, I have one question for you. You mentioned a number of different tumor types that historically have been brought up: benign mesotheliomas, solitary fibrous tumors, and others. Mesotheliomas and these fibrous tumors can easily be differentiated now by using immunohistochemical techniques. I was wondering whether you verified the difference between the malignant tumors that you removed and the 1 benign tumor that turned into a malignant tumor on second biopsy using immunohistochemical techniques.

Dr Harrison-Phipps. Thank you, Dr McFadden, for your comment and your question.

The study goes back to 1972, and therefore the first thing I would say is that not all these immunohistochemical techniques were available then, but in the majority of patients, the pathology reports did include immunocytochemistry, and the markers that were looked at were vimentin, cytokeratin, CD34, CD99, and Bcl-2. As you correctly point out, malignant mesothelioma is positive for cytokeratin but negative for these mesenchymal markers. The thing is that it does not necessarily help you with deciding which are benign and which are malignant. The only thing I can tell you is that there might be some future markers that could help predict recurrence, and one of these is called p16, which has been showing early promise at predicting recurrence with these solitary fibrous tumors.

Dr Michael Lanuti (Boston, Mass). I enjoyed your presentation.

One of the things that I and perhaps my colleagues struggle with is how to follow these patients. I would like your comments. I think that a malignant phenotype is a bit easier, and you would probably image them more frequently. In contrast, how often were patients with a benign phenotype screened during follow-up in your study? I struggle with how often one should image these patients because there is a small risk of malignant degeneration.

Dr Harrison-Phipps. Thank you very much for your question.

I think I would come back to the sensitivity of the imaging. Amazingly, chest radiographic analysis showed a sensitivity of 
$92 \%$, and CT scanning showed a sensitivity of $98 \%$. Therefore what I would say is that for patients with malignant tumors, they ought to be followed up for certain with a CT scan. I have some data on the recurrences and when they recurred. Of the 6 patients who experienced recurrence, the median time to recurrence was 37 months. Therefore you are predicting an early recurrence. The range of that was quite significant. It was actually from 12 to 132 months. Therefore there was a wide range. I guess the bottom line is that you have to be very vigilant at all times. It is very difficult to give you an exact answer, but I would certainly think that an annual or at least a 6-month CT scan would be essential for patients with malignant solitary fibrous tumors. I think it is easier for patients with benign tumors. You know, the risk of transformation from a benign tumor to a malignant tumor is low, and personally, I think an annual CT scan would be advisable. For people who have health care economic issues, a chest radiographic scan is almost as good at $92 \%$ sensitivity, but I certainly think at least an annual follow-up would be necessary for benign tumors and perhaps a 6-month follow-up for malignant tumors.

Dr Jhingook Kim (Seoul, South Korea). We have reported about 50 cases of our experience about 4 years ago. I have 3 questions or comments about how to approach these patients.

First of all, in my experience we do not have many patients who require anatomic lung resection. This is the case for the SFTPs. In most of the patients, we need just a wedge resection or otherwise just to catch the tumor from the pleural cavity. In your report you have so many pneumonectomies and lung resections.

Second, if the tumor is not so big, it is quite easy to just catch it. However, if the tumor is fairly big, we can resect the tumor portion by portion using small incisions. Because most of the fibrous tumors are benign, there is no need to approach with such a big incision, including the hemiclamshell.

My third question is this: What do you think about the need for neoadjuvant treatment if the tumor looks malignant or if the FNA shows the malignancy?

Thank you for your attention.

Dr Harrison-Phipps. Thank you very much, Dr Kim.

With regard to your first question, in so many lung resections the majority of the tumors are pedunculated, they arise from the visceral pleura, and they are on a stalk. I think the number of lung resections was probably around 10 or less in total. The majority of our excisions were wedge excisions. Seventy percent were wedge excisions. Therefore I concur with what you say. But I would caution you, because the bottom line is that you do not actually know the pathology until you remove the specimen. One of our predecessors, Dr Bernatz, has come up with a very useful comment, and he basically advises to consider the advice of the professional golfer to the weekend golfer: do not underclub. Do not underestimate these tumors. You have got to be careful. You want to take a little bit extra, if you can.

With regard to your second question concerning no need for large incisions for larger tumors, do I understand that you remove these piecemeal? I was not quite sure what you were meaning there. I understand you remove them bit by bit.
To come back to the first comment, I certainly think the posterolateral thoracotomy is very useful for large tumors. You really want to get all the tumor out, if you can. You are aiming for a clear resection margin. This is what the large case series of 232 patients by England and colleagues supported. You are quite right to suggest that the majority are very easy operations, some even using VATS. It is a polypoid tumor that is easy to remove. But we have to be careful with the larger ones because there is a risk of recurrence, even with benign tumors, and the larger the tumors, obviously they could be malignant, and I really think that it is important to think about your operative approach. In most cases a posterolateral thoracotomy would be fine, but perhaps there are arguments for more extended operations as well.

As for the need for neoadjuvant treatment, I do not know whether we will ever get to know this. First of all, the tumors are very rare. A preoperative diagnosis is hard to establish, certainly for malignant tumors. You would never get it on the basis of an inadequate or uncertain report, and it is really unusual for an FNA to diagnose a malignant SFTP preoperatively. Therefore I am not sure we will ever see many patients who have had neoadjuvant treatment.

Dr Jean Deslauriers (Quebec City, Quebec, Canada). This is an academic question really. These tumors classically are associated with paraneoplastic syndromes, such as clubbing and hypoglycemia. In fact, the classic presentation is that it is a big tumor with clubbing. Maybe you mentioned it in your presentation, but I did not hear it. What percentage of these patients really had one or the other classic paraneoplastic syndromes, such as clubbing, and does this have an effect on whether the tumor is benign at the end of the day or malignant? Because this is one of the largest, if not the largest, series of such tumors, I would be interested to know the exact incidence of these paraneoplastic syndromes, if you were able to look at that aspect of these tumors.

Dr Harrison-Phipps. Thank you very much for your question, Dr Deslauriers.

The incidence of hypertrophic pulmonary osteoarthropathy in this series was 8 patients, or $9.9 \%$; the incidence of persons with clubbing was 3 patients; and the incidence of hypoglycemia was 1 patient. You quite rightly mentioned these names, and these names have syndromes in the literature. Hypoglycemia is known as the Doege-Potter syndrome and hypertrophic pulmonary osteoarthropathy, and clubbing is known as the Pierre-Marie-Bamberg syndrome. In our series it was $10 \%$ for these symptoms. That is pretty much what you can find in the other series as well. There are 1 or 2 series with more or less, but really, in our series, of those 8 patients or 9 with hypoglycemia who had these symptoms, only 2 were malignant. Therefore I do not really think we can say it predicts malignancy. It is simply due to the fact that for osteoarthropathy, they might have an excess production of hyaluronic acid, and for hypoglycemia, they have an excess production of insulin 2 growth-like factor. It is difficult to say. I do not necessarily think it is related to size or malignancy. It is just a very interesting feature of these unusual tumors. 